\title{
EFECTO DE FITOREGULADORES ENRAIZANTES Y LATEMPERATURA EN EL ENRAIZAMIENTO DE ESTACAS DE Myrciaria dubia (HBK) MC VAUGH, CAMU CAMU ARBUSTIVO, EN UCAYALI-PERÚ
}

\author{
EFFECT OF ROOTERS FITOREGULATORS AND THE TEMPERATURE IN STAKE-ROOTING \\ OF Myrciaria dubia (HBK) MC VAUGH, ARBUSTIVE CAMU CAMU, IN UCAYALI - PERU
}

\section{Carlos Alberto Oliva Cruz ${ }^{1}$}

\section{RESUMEN}

En la Estación Experimental del IIAP en Ucayali, entre los meses de octubre de 2003 y enero de 2004, se instaló la prueba de enraizamiento, utilizando ácido indolbutírico (AIB) y ácido naftalenacético (ANA) como hormonas de enraizamiento, bajo un diseño de bloques completamente al azar (DBCA), con cuatro tratamientos, un testigo y tres repeticiones; considerando 15 estacas por tratamiento. Los tratamientos fueron: 200 ppm de AIB con 24 horas de inmersión, 200 ppm de AIB con 48 horas, 200 ppm de ANA con 24 horas y 200 ppm de ANA con 48 horas de inmersión y un testigo sin aplicación. Se evaluaron las variables número y longitud de brotes, número y longitud de raíces y porcentaje de enraizamiento. A los 30 días no se encontró diferencias significativas en la variable $\mathrm{n}^{\mathrm{o}}$ y longitud de brotes, pero el mejor comportamiento se observó en el tratamiento testigo con $4.5 \mathrm{~cm}$ y 4.73 en longitud y número de brotes respectivamente; en relación a las demás variables sí encontramos diferencias significativas (Tukey $0.05 \%$ ), considerando como mejor tratamiento a 200 ppm AIB con 48 horas de inmersión, seguido por 200 ppm AIB con 24 horas de inmersión con 80 y 60\% de enraizamiento, 5.13 y 2.33 en número de raíces, 4.56 y $2.55 \mathrm{~cm}$ en longitud de raíces respectivamente.

Palabras clave: fitorreguladores, ácido, inmersión, enraizamiento y estacas.

\section{ABSTRACT}

In IIAP`s Experimental Station, in Ucayali, from October 2003 to January 2004, this test was setup, using indlebutyric acid (AIB) and sour acetic naftalena (ANA) as enraizamiento hormones, under a Design of Blocks Totally at random (DBCA), with 4 treatments: one witness and 3 repetitions; at 15 stakes for treatment. The treatments were: 200 ppm of AIB with 24 hours of immersion, 200 ppm AIB at 48 hours, 200 ppm ANA with 24 hours and 200 ppm ANA with 48 hours of immersion and a witness without application. The variable number and longitude of buds, number and longitude of roots and enraizamiento percentage were evaluated. To the 30 days he/she was not significant differences in the variable $\mathrm{n}^{\circ}$ and longitude of buds, but the best behavior one observes in the treatment witness with $4.50 \mathrm{~cm}$ and 4.73 in longitude and number of buds respectively; in relation to the other variables if we find significant differences (Tukey 0.05\%) whereas clause as better treatment to $200 \mathrm{ppm}$ AIB with 48 hours of immersion, continued by 200 ppm AIB with 24 hours of immersion with 80 and 60 enraizamiento\%, 5.13 and 2.33 in number of roots, 4.56 and $2.55 \mathrm{~cm}$ in longitude of roots respectively.

Key words: fitoregulador, indolebutyric acid, myrciaria dubia, rooting, camu camu, stakes.

\section{INTRODUCCIÓN}

El camu camu arbustivo, es una especie silvestre, cuya explotación comercial es aún incipiente. En las poblaciones naturales encontradas en las riberas de los ríos amazónicos, se observa gran variabilidad fenotípica en sus diversas características. Actualmente para trabajos de propagación, se está empleando principalmente el método de injerto. Sin embargo el injerto no es el más indicado para la propagación de plantas madre en el trabajo de mejoramiento, por la probable influencia entre el patrón y la yema sobre la calidad genética o la expresión

1 Ingeniero agrónomo, Instituto de Investigaciones de la Amazonía Peruana-IIAP/ Correo electrónico: coliva@iiap.org.pe 
fenotípica. En cambio en la propagación por estacas, se logra una réplica del ADN, es decir toda la información genética de la planta madre, según Vásquez (2000).

Desde 1978, se efectuaron varios ensayos intentando enraizar estacas de distintos diámetros, con variados sustratos y fitohormonas. Utia \& Pinedo (1979), ensayaron el enraizamiento, utilizando tres sustratos (arena, tierra y aserrín), en ninguno de los casos obtuvieron enraizamiento. En la Estación Experimental San Roque, realizaron un estudio de propagación, donde se utilizaron estacas con y sin talón, con temperatura y humedad controlada con tratamiento obteniendo cero porciento de enraizamiento.

La dificultad del enraizamiento de alguna forma fue explicada por Hartman \& Kester (1999), quien afirma que la capacidad de formar raíces disminuye con el aumento de la edad de las plantas y que la tierra debe ser ligera, suelta y convenientemente húmeda. A esto, Lorente (1999) agrega que, con la aplicación de técnicas como: hormonas, nebulización, humidificación y calentamiento basal, se favorece y se aumenta la radicación de las estacas. Prueba de ello, Menezes (1998) alude los buenos resultados en la propagación por estaca del camu camu, quien utilizó arena y aserrín como sustratos y 00, 150, 300, 1000 y 1500 ppm de ácido indolbutírico, obteniendo sobre sustrato de arena hasta 73\% de enraizamiento en las concentraciones de 300 y 1000 ppm de AIB.

Vásquez (2000), logró en estacas de camu camu, 0, 10 y 20\% de enraizamiento para los diámetros 0,5; 2 y >2,5 cm, respectivamente; la posición de las estacas sobre el sustrato fue inclinada y los riegos aplicados diariamente.

En el INPA (Brasil) Santana (1997), realizó enraizamiento, utilizando como sustratos arena y aserrín, mediante la aplicación de hormonas enraizantes con dosis de 0, 200, 2000, 20000 ppm de ANA y toque al 20\% de ANA, bajo cuatro riegos diarios a través de 4 aspersores; logró obtener los mejores resultados en estacas tratadas con 200 y 2000 ppm de ANA con 56 y 48\% respectivamente y no encontró diferencias significativas entre sustratos.

Galucio, P.B. (2002), utilizando estacas con diámetros mayores que 0,8 mm de diámetro y aplicando 200 ppm de ácido naftalenacético (ANA), logró obtener a los 90 días hasta 90\% de enraizamiento.

En ese sentido, conociendo la importancia de la especie y la propagación por estacas, el presente trabajo plantea utilizar fitohormonas enraizantes con la finalidad de evaluar la eficiencia en el enraizamiento de estacas de camu camu.

\section{MATERIAL Y MÉTODO}

Entre los meses de octubre de 2003 y enero de 2004, en la EE-IIAP Ucayali, se instaló el ensayo de enraizamiento de estacas de camu camu. La ubicación georeferencial esta dada en las coordenadas de $8^{\circ} 22^{\prime}$ '31" de latitud sur, 74 34' 35" de longitud oeste; altitud de $154 \mathrm{msnm}$; temperatura máxima promedio de $34,4^{\circ} \mathrm{C}$, promedio mínimo de $20,2{ }^{\circ} \mathrm{C}$, y una humedad relativa de $82.93 \%$.

Se emplearon estacas de $25 \mathrm{~cm}$ de longitud y 1,5 a 2,0 cm de diámetro, provenientes de las ramas laterales de plantas de nueve años en desarrollo vegetativo. Las estacas fueron instaladas en posición vertical en camas (3 x 1 x 0,2 m) con arena como sustrato. La cama fue cubierta con plástico transparente a 1,5 m de altura en cuyo interior se ubicó un termómetro ambiental marca Brand para el control de la temperatura ambiental interna. El trabajo de riego estaba en función al comportamiento de la temperatura, siendo frecuente cuando se excedía los $40^{\circ} \mathrm{C}$, por espacio de 5 a 6 minutos por riego.

La instalación fue realizada en bloques completamente al azar, con cinco tratamientos y tres repeticiones; un testigo, 200 ppm de AIB con 24 horas, 200 ppm de AIB con 48 horas, 200 ppm de ANA con 24 horas y 200 ppm de ANA con 48 horas de inmersión, llegando a constituirse 15 estacas tratamiento.

En el presente trabajo, utilizamos como fitorreguladores, el ácido naftalenacético (ANA) y el ácido indolbutírico (AIB). El tercio laboral de estacas fue según los tratamientos. 
Las evaluaciones se realizaron a los 60 y 90 días de la instalación, donde se evaluó: número y longitud de brotes, número y longitud de raíces y porcentaje de enraizamiento. Los datos obtenidos fueron procesados y analizados utilizando el programa estadístico SPSS versión 10.07.

\section{RESULTADOS}

Los brotes en las estacas presentaron similar comportamiento para todo los tratamientos, existiendo unos mejores que otros, pero lejos de existir diferencias significativas.

Tabla 1. Longitud y número de brotes, en estacas de camu camu, mediante la aplicación de fitorreguladores enraizantes en la EE-IIAP Ucayali.

\begin{tabular}{|c|c|c|c|c|c|c|}
\hline Variable & Tratamiento & $\mathbf{N}$ & Promedio & $\begin{array}{c}\text { Desviación } \\
\text { estandar }\end{array}$ & Mínimo* & Máximo* \\
\hline \multirow[t]{5}{*}{ Long. brotes } & Testigo & 3 & 4.50 & 2.99 & 2.30 & 7.90 \\
\hline & 200 ppm AIB -24 hr & 3 & 1.87 & 1.72 & 0.00 & 3.40 \\
\hline & 200 ppm AIB - 48 hr & 3 & 3.80 & 1.83 & 1.80 & 5.40 \\
\hline & 200 ppm ANA - 24 hr & 3 & 1.93 & 1.45 & 0.50 & 3.40 \\
\hline & 200 ppm ANA - 48 hr & 3 & 1.40 & 0.56 & 0.90 & 2.00 \\
\hline \multirow[t]{5}{*}{ N. ${ }^{\circ}$ brotes } & Testigo & 3 & 4.73 & 2.20 & 3.00 & 7.20 \\
\hline & 200 ppm AIB -24 hr & 3 & 2.47 & 1.03 & 1.60 & 3.60 \\
\hline & 200 ppm AIB - 48 hr & 3 & 3.33 & 2.12 & 1.40 & 5.60 \\
\hline & 200 ppm ANA - 24 hr & 3 & 2.33 & 0.93 & 1.80 & 3.40 \\
\hline & 200 ppm ANA - 48 hr & 3 & 2.67 & 1.67 & 0.80 & 4.00 \\
\hline
\end{tabular}

* Evaluación realizada a los 30 días.

El testigo presentó mejor resultado, tanto en número como en longitud de brotes con 4,73 y 4,5 cm respectivamente (tabla 1). Con respecto a los tratamientos con fitohormonas enraizantes, los resultados fueron mucho más homogéneos con ligera superioridad para 200 ppm de AIB-48 horas con 3.33 brotes y $3.80 \mathrm{~cm}$ de longitud. Esto hace suponer, que el tratamiento con hormonas de alguna forma inhibe la emisión de brotes, coincidiendo con Angosto T.T.(2002), quien sustenta que las auxinas hormonas regulan la dominancia apical.

El enraizamiento de las estacas durante los 60 y 90 días, experimenta comportamiento heterogéneo, observando efectos significativos de las hormonas en la formación de raíces. En efecto, el ácido indolbutírico (AIB) tiene mejor resultado llegando a los 90 días hasta el 80\% de enraizamiento (tabla 2 y figura 1). El efecto de esta hormona hasta los 60 días en ambos tiempos de inmersión (24 y 48 horas) tiene comportamiento similar con 33.3\%; luego la superioridad se hace evidente para 48 horas de inmersión cuando prolongamos hasta los 90 días logrando incrementar 46.7\%; estos resultados hacen referencias de la exigencia de una regresión directa positiva, considerando que a mayor tiempo de inmersión tendremos mejores resultados. 
Tabla 2. Porcentaje de enraizamiento de estacas de camu camu, a los 60 y 90 días después de instalado el experimento en el IIAP Ucayali.

\section{$\%$ enraizamiento}

\begin{tabular}{lccccc}
\hline Tratamiento & $\mathbf{N}$ & $\begin{array}{c}\text { Promedio } \\
\text { (60 días) }\end{array}$ & $\begin{array}{c}\text { Desviación } \\
\text { estandar }\end{array}$ & $\begin{array}{c}\text { Promedio } \\
\text { (90 días) }\end{array}$ & $\begin{array}{c}\text { Desviación } \\
\text { estandar }\end{array}$ \\
\hline Testigo & 3 & .00 & .00 & 20.00 & 20.00 \\
200 ppm AIB-24hr & 3 & 33.33 & 11.55 & 60.00 & .00 \\
200 ppm AIB-48 hr & 3 & 33.33 & 11.55 & 80.00 & .00 \\
200 ppm ANA-24 hr & 3 & 33.33 & 11.55 & 40.00 & .00 \\
200 ppm ANA-48 hr & 3 & .00 & .00 & 20.00 & 20.00 \\
\hline
\end{tabular}

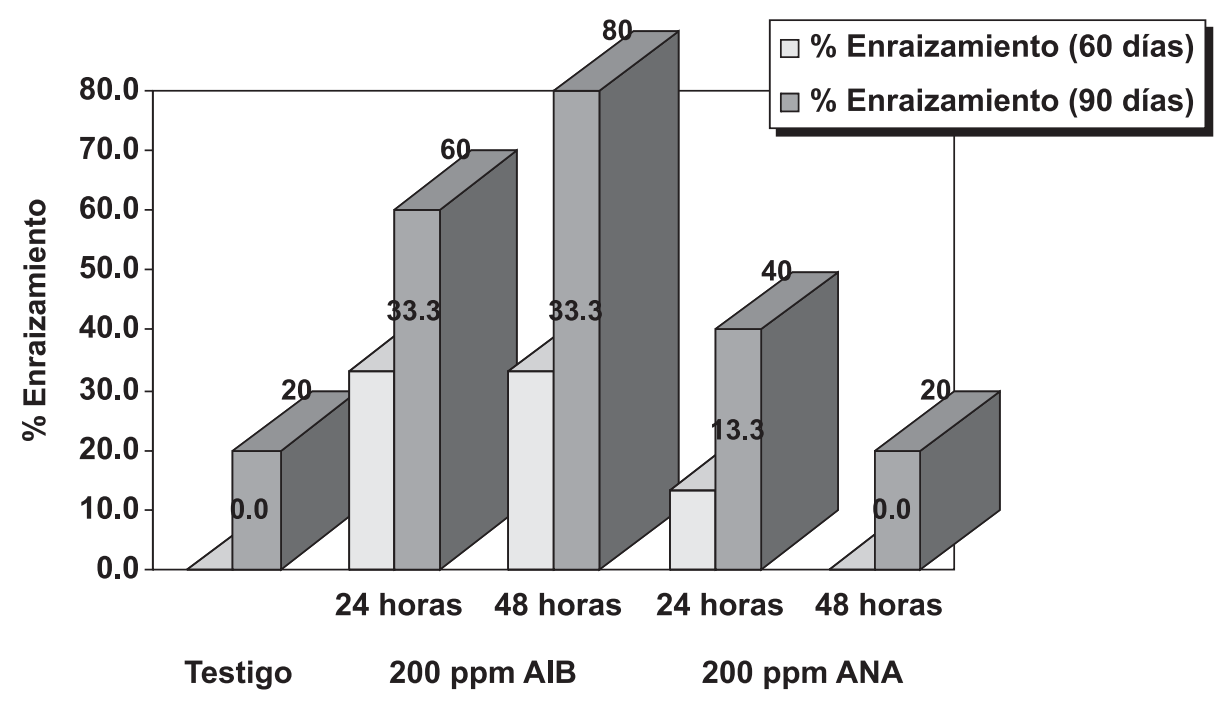

Figura 1. Enraizamiento de estacas de camu camu a los 60 y 90 días de instalado el experimento.

Sin embargo, el efecto del ácido naftalenacético (ANA), en la formación de raíces no muestra eficiencia bajo estas condiciones, sólo se logró obtener hasta 40\% de enraizamiento en 24 horas de inmersión y 20\% para 48 horas de inmersión, lo que hace notar un comportamiento inverso al AIB.

La tendencia en los niveles de enraizamiento logrado con ANA es inversa al obtenido con AIB. A mayor tiempo de inmersión en la solución de ANA el porcentaje de enraizamiento fue menor, y similar al tratamiento testigo. Esto pone en evidencia que el ANA no es muy efectivo, contradictorio a lo mencionado por Santana, (1997) y Galucio, P. B . (2002), ambos aducen haber encontrado resultados satisfactorios utilizando 200 ppm AIB hasta 56 y $90 \%$ respectivamente.

Cuando las estacas de camu camu, carecen de tratamiento hormonal, la formación de raíces es significativamente menor (tabla 2), esto se observa en el testigo donde se logró hasta $20 \%$ de enraizamiento, resultado que concuerda con lo reportado por Vásquez (2000) y de alguna forma se ha comportado de manera similar cuando se aplicó 200 ppm de ANA con 48 horas de inmersión. 
Tabla 3. Longitud y número de raíces, en estacas de camu camu, mediante la aplicación de enraizantes en la EE-IIAP Ucayali.

\begin{tabular}{lllcccc}
\hline Variable & \multicolumn{1}{c}{ Tratamiento } & N & Promedio & $\begin{array}{c}\text { Desviación } \\
\text { estandar }\end{array}$ & Mínimo* & Máximo* \\
\hline Long. de raíces & Testigo & 3 & 0.87 & 1.18 & 0.00 & 2.20 \\
& 200 ppm AIB -24 hr & 3 & 2.55 & 1.08 & 1.30 & 3.24 \\
& 200 ppm AIB - 48 hr & 3 & 4.56 & 0.10 & 3.42 & 5.26 \\
& 200 ppm ANA - 24 hr & 3 & 1.59 & 0.73 & 1.04 & 2.42 \\
& 200 ppm ANA - 48 hr & 3 & 0.87 & 1.50 & 0.00 & 2.60 \\
\hline N. ${ }^{\circ}$ de Raíces & Testigo & 3 & 0.67 & 0.99 & 0.00 & 1.80 \\
& 200 ppm AIB -24 hr & 3 & 2.33 & 0.81 & 1.40 & 2.80 \\
& 200 ppm AIB - 48 hr & 3 & 5.13 & 2.89 & 1.80 & 6.80 \\
& 200 ppm ANA - 24 hr & 3 & 0.80 & 0.53 & 0.40 & 1.40 \\
& 200 ppm ANA - 48 hr & 3 & 0.73 & 0.64 & 0.00 & 1.20 \\
\hline
\end{tabular}

Por otro lado, en relación a longitud y número de raíces (tabla 3), observamos que las estacas tratadas con AIB, muestran superioridad, logrando en promedio 2,55 y 4,56 cm de longitud en la raíz, resultado mayor para las estacas con 48 horas de inmersión en 200 ppm de AIB. También encontramos excelentes resultados con respecto al número de raíces estaca, logrando en promedio hasta 5,13 raíces. La variable número de raíces es determinante en el éxito del establecimiento de las estacas enraizadas en campo definitivo. Estacas deficientes en raíz y de corta longitud presentan problemas de prendimiento llegando muchas veces a una muerte paulatina, debido a la baja capacidad de fijar nutrientes.

Tabla 4. Prueba estadística de Tukey en el enraizamiento de estacas de camu camu, mediante la aplicación de enraizantes en la EE-IIAP Ucayali.

\begin{tabular}{lccccc}
\hline Tratamiento & $\begin{array}{c}\% \\
\text { enraizamiento }\end{array}$ & $\begin{array}{c}\mathbf{N}^{\mathbf{0}} \\
\text { raíces }\end{array}$ & $\begin{array}{c}\text { Longitud } \\
\text { de raíz }\end{array}$ & $\begin{array}{c}\mathbf{N}^{\mathbf{0}} \mathbf{d e} \mathbf{d e} \\
\text { brotes* }\end{array}$ & $\begin{array}{c}\text { Longitud de } \\
\text { brotes* }\end{array}$ \\
\hline Testigo & $20.00 \mathbf{c}$ & $0.67 \mathbf{b}$ & $0.87 \mathbf{b}$ & $4.73 \mathbf{a}$ & $4.50 \mathbf{a}$ \\
200 ppm AIB -24 hr & $60.00 \mathbf{a b}$ & $2.33 \mathbf{a b}$ & $2.55 \mathbf{b}$ & $2.47 \mathbf{a}$ & $1.87 \mathbf{a}$ \\
200 ppm AIB - 48 hr & $80.00 \mathbf{a}$ & $5.13 \mathbf{a}$ & $4.56 \mathbf{a}$ & $3.33 \mathbf{a}$ & $3.80 \mathbf{a}$ \\
200 ppm ANA - $24 \mathrm{hr}$ & $40.00 \mathbf{b c}$ & $0.80 \mathbf{b}$ & $1.59 \mathbf{b}$ & $2.33 \mathbf{a}$ & $1.93 \mathbf{a}$ \\
200 ppm ANA - 48 hr & $20.00 \mathbf{c}$ & $0.73 \mathbf{b}$ & $0.80 \mathbf{b}$ & $2.67 \mathbf{a}$ & $1.40 \mathbf{a}$ \\
\hline
\end{tabular}

* Evaluación realizada a los 30 díasMedias seguidas de la misma letra en vertical, no difieren entre sí al 5\% de probabilidad, por la prueba de Tukey. 
Según la prueba estadística de Tukey (tabla 4), se atribuye los mejores resultados a las estacas tratadas con AIB en sus dos tiempos de inmersión de 48 y 24 horas, con 80 y 60\% de enraizamiento respectivamente y en número de raíces con 5,13 y 2,33 raíces estaca; ambos tratamientos se comportan estadísticamente iguales en estas variables, pero diferentes en longitud de raíces con 4,56 y 2,55 cm respectivamente; y en relación al número y longitud de brotes no encontramos diferencias significativas.
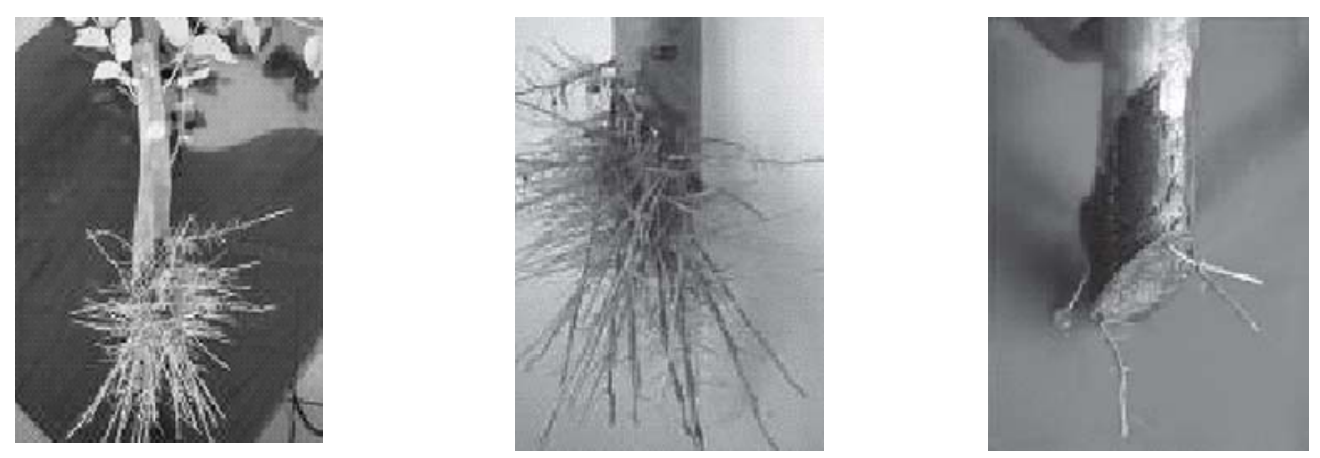

Las fotos de izquierda y centro responden al enraizamiento con aplicación de hormonas y la foto de la derecha es una estaca enraizada del tratamiento testigo (sin hormonas).

\section{CONCLUSIONES}

- El ácido indolbutírico, presentó mayor eficiencia en el enraizamiento de estacas de camu camu, en comparación a los resultados obtenidos con el ácido naftalenacético.

- Las estacas de camu camu tratadas con el ácido indolbutírico, en mayor tiempo de inmersión presenta mejores resultados. Resultados que se sustentan en un alto porcentaje de enraizamiento y un alto número y longitud de raíces.

- Las estacas de camu camu con mayor tiempo de inmersión en solución de ácido naftalenacético, presenta menor resultado, expresando bajos valores en número y longitud de raíces.

- La utilización del plástico transparente, ha permitido incrementar la temperatura interna de la cama, promoviendo la rapidez en las reacciones químicas y por ende la acción de las hormonas en la formación de raíces.

- La utilización del termómetro dentro de la cama fue fundamental, por que facilitó monitorear la temperatura, lográndose determinar que a mayor de $40{ }^{\circ} \mathrm{C}$ los brotes tienden a secarse paulatinamente.

- Las estacas de camu camu cuando no son tratadas con hormonas de enraizamiento (testigo) presentan dificultad para enraizar; del bajo porcentaje que se obtiene, resultan estacas con reducido número de raíces y corta longitud.

\section{RECOMENDACIONES}

- Para trabajos posteriores, se recomienda considerar mayores tiempos de inmersión sólo para AIB, con la finalidad de establecer un tiempo adecuado de inmersión de las estacas.

- Para otros trabajos, se debe tomar en consideración un mes más de evaluación, proyectándolos hasta los 120 días.

- Se recomienda realizar un nuevo experimento bajo estas mismas condiciones con la finalidad de validar el presente trabajo. 


\section{BIBLIOGRAFÍA}

ANGOSTO T. T. 2002. Reguladores de crecimiento vegetal y biotecnología. Folleto del curso realizado en la Universidad Nacional Agraria La Molina, desde el 17 hasta el 19 de octubre de 2002.

BAOS, C.P.; B.F.D.FLOR y C.P. TRUEBA. 1986. Descriptores de camu camu. Programa Nacional de Cultivos Tropicales. (Informe Técnico, n. ${ }^{\circ}$ 8). 55 p.

ENCISO, R. 1992. Propagación de camu camu (Myrciaria dubia) por injerto. Informe Técnico n. 18. Programa de Investigación en Cultivos Tropicales-INIA. Lima. 17 p.

FLORES, P. S. 1997. Cultivo de frutales nativos amazónicos. Manual para extensionistas. TCA. Lima-Perú. 307 p.

GALUCIO, P. B. 2002. Producción de mudas de camu camu (Myrciaria dubia (H.B.K.) Mc Vaugh) por estacas utilizando ramas provenientes de diferentes tipos y posiciones de la planta.Nota Técnica. INPA-Brasil.

HARTMANN \& KESTER. 1999. Propagación de Plantas. 7tma Reimpresión. Editorial Continental. México. Págs. 219-224, 255-303, 319-331, 336-340, 348-350, 352, 355-358.

HUDSON, T. H. y E. K. DALE. 1995. Propagación de plantas. Editorial Continental. México. 733 p.

LORENTE, H. J. 1999. Biblioteca de la Agricultura. Editorial LEXUS. Pág. 130-131.

LÓPEZ A.U. 2002. Aplicación de niveles de calcio, boro, cobre y zinc, sobre la productividad del camu camu en suelos aluviales. (Informe trimestral 2003)-IIAP-Ucayali.

MENEZES, D. 1998. Efeitos de diferentes reguladores de crescimento sobre o enraizamento de estacas de camucamu (Myrciaria dubia (H.B.K.) Mc Vaugh). FCA/UA. Brasil. 2 Pág.

SANTANA, S. 1998. Propagação de Camu-Camu (Myrciaria dubia (H.B.K.) Mc Vaugh), por meio de estaquia. BIO*Tecnología. www.biotecnologia.com.

UTIA Y PINEDO (1979), Propagación del Arazá y camu camu. Tésis presentada para optar el titulo de Ingeniero Agrónomo. Universidad Nacional de la Amazonia Peruana. Iquitos-Perú.

VÁSQUEZ MATUTE A. (2000) «El Camu camu, Cultivo, Manejo e Investigaciones». Universidad Nacional de la Amazonía Peruana- Facultad de Agronomía- Iquitos-Perú. 